\title{
Interferon-Alpha 2-a and Its Dual Effect in Treating Two Diseases (Hepatitis C and Polycythemia Vera)
}

\author{
Hawraa Shwaylia ${ }^{a}$ Elrazi Ali ${ }^{b}$ Mohamed A. Yassin ${ }^{a}$ \\ aDepartment of Medical Oncology, Hamad Medical Corporation, Doha, Qatar; \\ bInternal Medicine Department, Hamad Medical Corporation, Doha, Qatar
}

\section{Keywords}

Polycythemia vera $\cdot$ Hepatitis C $\cdot$ Hepatitis $\cdot$ Interferon

\begin{abstract}
Hepatitis $C$ and polycythemia vera (PV) co-existence is not rare; it has been reported in the literature. Treatment with interferon (IFN) has been used to treat both conditions; however, the use of IFN in concomitant hepatitis C infection with PV and its outcome are rarely described in the literature. Here, we report a 56 -years-old male patient with hepatitis $C$ virus infection and PV, who was treated with IFN for his chronic hepatitis $C$, which resulted in significant improvement of $\mathrm{HB}$ as well as normalization of his bone marrow and eradication of the clone (Jak2 V617F).
\end{abstract}

\section{Introduction}

Myeloproliferative neoplasms (MPNs) are hematological disorders distinguished by increased proliferation and buildup of mature myeloid cells in the bone marrow and peripheral blood. MPNs are classified as Philadelphia chromosome-positive or -negative [1]. The classic Philadelphia-negative MPNs include essential thrombocythemia, polycythemia vera (PV), and primary myelofibrosis [2]. They are usually sporadic; however, familial cases have been described in different parts of the world [3]. Several methods have been utilized in the treatment of MPN, such as cytoreduction, JAK 2 inhibitors, and interferon (IFN). In 1957, IFN was discovered by Isaacs and Lindenmann. IFNs are glycoproteins related to cytokines and have antitumor antiviral and immunomodulatory activities. There are 2 types of IFNs: type I and type II; type I IFNs includes IFN $\alpha$ and IFN $\beta$. Type II IFN constitutes solely of IFN $\gamma$. Each type has unique genetic derivation, primary producing cells, and properties [4]. In the field of 
viral interference, it was believed that viral interference had been considered to be due to the direct action of one virus on the pathologic activity of a second agent, but later on, it was shown to be proteins that in turn activate several genes responsible for the biologic effects imputed to the IFNs in addition to their antiviral activity [5]. Nowadays, IFN is used widely in medical practice; it is used to treat neoplastic diseases, viral infections, and myeloproliferative disorders [4].

\section{Case Report}

The case is a fifty-six-year-old Egyptian gentleman diagnosed with hepatitis C virus infection in 2011 based on serology. Hepatitis C viral load was 2458678, genotype 4. Concomitantly, he was diagnosed with PV based on the WHO criteria 2008 due to persistently elevated hemoglobin level, above $18.5 \mathrm{gm} / \mathrm{dL}$ (13-17 g/dL). Further investigations showed Jak2 was positive with a low erythropoietin level. The patient received pegylated IFN $\alpha 2 \mathrm{a}$ in a dose of $180 \mathrm{mcg} /$ week for 5 years. It took 2 years for the complete eradication of hepatitis C. He was cured of hepatitis c virus, and hemoglobin levels were normal (14-15.5 gm/dL), and repeated Jak $2 \mathrm{v} 617 \mathrm{f}$ was negative. Bone marrow biopsy was done to evaluate for PV at the bone marrow level (panmyelosis). The bone marrow reported normal (no features suggestive of PV or other MPNs).

\section{Discussion}

Chronic inflammation and immunoregulation are likely to be involved in the pathogenesis of MPNs. Thereby, high levels of circulating inflammatory cytokines have been found and have prognostic value. Chronic inflammation diminishes IFN signaling, and from this point, the efficacy of IFN in MPNs was recognized [6].

IFN has an expanded range of biological and molecular activities, including cytotoxicity, tumor-promoting immune cell populations, activation of multiple proapoptotic genes and proteins, and downregulation of several antiapoptotic proteins. This component makes IFN an agreeable therapeutic agent in patients with cancer in general and in patients with MPNs in specific. IFN emphasizes special activities that support its use in MPNs, including PV. IFNs have direct inhibition of bone marrow fibroblast progenitor cells and suppression of hematopoietic progenitor proliferation, which results in controlling erythrocytosis and thrombocytosis [7]. In 1993, the therapeutic benefit described by Silver, followed by many studies, demonstrated the use of IFN $\alpha$ therapy for PV. IFN $\alpha$ therapy benefits include the induction of hematologic remission (with the elimination or reduction in the need for phlebotomy), control of thrombocytosis and leukocytosis, resolution of disease-associated symptoms (particularly refractory pruritus), and resolution of splenomegaly [8].

In 1991, IFN was first approved for the treatment of hepatitis C. It was introduced to treat chronic hepatitis $\mathrm{C}$ as IFN $\alpha$, then improved by pegylation form, and then the addition of ribavirin resulted in a better sustained virologic response. At present, the knowledge of HCV complexity and character resulted in the evolution of direct-acting antivirals and IFN-free era. IFNs have 2 mechanisms of actions in treating hepatitis C: first, by binding to cell-surface receptors that signal through the system of Janus-activated kinase and signal transducers and activators of transcription, resulting in induction of numerous IFN-stimulated genes (doublestranded RNases, inhibitors of viral protein translation, and proteins that destabilize viral messenger RNA). The second mechanism is by modulating gene expression involved in the immune response, leading to activation of natural killer cells, maturation of dendritic cells, the proliferation of memory T cells, and prevention of T-cell apoptosis [9].

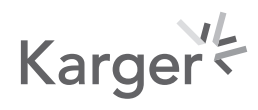


Hepatitis $\mathrm{C}$ is a silent disease, and $80 \%$ develop into a chronic infection. Chronically infected patients need to be treated with antiviral due to the high risk of progression of liver damage to cirrhosis or carcinoma if left without treatment. Treatment aims to reach a sustained virologic response, which means undetectable viral RNA over 6 months after treatment completion. The second goal of the therapy is to improve the quality of life and prevent progression to hepatocellular carcinoma [10]. Treatment is indicated for individuals with positive anti-HCV, HCVRNA-positive patients with increased serum aminotransferase levels, and for whom there is histological evidence of chronic hepatitis. Some considerations should be considered when deciding to initiate treatment, which includes age, comorbidities, extrahepatic manifestations such as cryoglobulinemia and glomerulonephritis, and quality of life [11, 12].

\section{Conclusion}

For patients with concomitant PV and hepatitis $\mathrm{C}$ virus infection, IFN2 $\alpha$ is the preferred antiviral cytoreductive agent due to its dual effect.

\section{Acknowledgement}

The authors wish to show my gratitude to the Internal Medicine Residency program, to Dr. Dabia Hamad Almohanadi, and to Qatar National Library for the scientific support. And the authors would like to thank the patient for allowing us to share his case.

\section{Statement of Ethics}

Written informed consent was obtained from the patient for publication of this case report. The case was approved by the medical research center (MRC-04-20-953).

\section{Conflict of Interest Statement}

All authors have no conflict of interest.

\section{Funding Sources}

Qatar National Library.

\section{Author Contributions}

Hawraa Shwaylia: writing, editing, and final approval. Elrazi Ali: writing, editing, and final approval. Mohamed Yassin: writing, editing, and final approval.

\section{Karger'}




\section{References}

1 Yassin MA, Taher A, Mathews V, Hou HA, Shamsi T, Tuğlular TF, et al. MERGE: a multinational, multicenter observational registry for myeloproliferative neoplasms in Asia, including Middle East, Turkey, and Algeria. Cancer Med. 2020 Jul;9(13):4512-26.

2 Turkina A, Wang J, Mathews V, Saydam G, Jung CW, Al Hashmi HH, et al. TARGET: a survey of real-world management of chronic myeloid leukaemia across 33 countries. Br J Haematol. 2020 Sep;190(6):869-76.

3 Al-Dewik N, Ben-Omran T, Zayed H, Trujillano D, Kishore S, Rolfs A, et al. Clinical Exome Sequencing unravels new disease-causing mutations in the myeloproliferative neoplasms: a pilot study in patients from the state of Qatar. Gene. 2019 Mar 20;689:34-42.

4 Friedman RM. Clinical uses of interferons. Br J Clin Pharmacol. 2008 Feb;65(2):158-62.

5 Skov V, Riley C, Thomassen M, Kjær L, Larsen TS, Bjerrum OW, et al. Interferon-alfa2 treatment of patients with polycythemia vera and related neoplasms impacts deregulation of oxidative stress genes and antioxidative defence mechanisms. Potential implications of IFN-alfa induced changes in TP53, NRF2 and CXCR4 for genomic instability and CD34+ mobilisation. Blood. 2018 Nov 29;132(Suppl 1):4326.

6 Verger E, Cassinat B, Chauveau A, Dosquet C, Giraudier S, Schlageter MH, et al. Clinical and molecular response to interferon- $\alpha$ therapy in essential thrombocythemia patients with CALR mutations. Blood. 2015 Dec 10; 126(24):2585-91.

7 Nazha A, Khoury JD, Verstovsek S, Daver N. Second line therapies in polycythemia vera: what is the optimal strategy after hydroxyurea failure? Crit Rev Oncol Hematol. 2016 Sep 1;105:112-7.

8 Falchi L, Newberry KJ, Verstovsek S. New therapeutic approaches in polycythemia vera. Clin Lymphoma Myeloma Leuk. 2015 Jun 1;15 Suppl:S27-33.

9 Hoofnagle JH, Seeff LB. Peginterferon and ribavirin for chronic hepatitis C. N Engl J Med. 2006 Dec 7;355(23): 2444-51.

10 Valenti WM. Clinical guidelines for the medical management of hepatitis C. Albany, NY: New York State Department of Health; 2005.

11 Kershenobich D. Module IV Indications and contraindications for treatment of hepatitis C virus infections. Annals of Hepatology. 2006;5(S1):22-3.

12 Taher A, Yassin MA, Xiao Z, Hou H-A, Tuglular T, Mathews V, et al. Impact of myeloproliferative neoplasms (MPNs) on health-related quality of life (HRQOL) and medical resource utilization: results from the MERGE registry. Blood. 2018 Nov 29;132(Suppl 1):4311. 